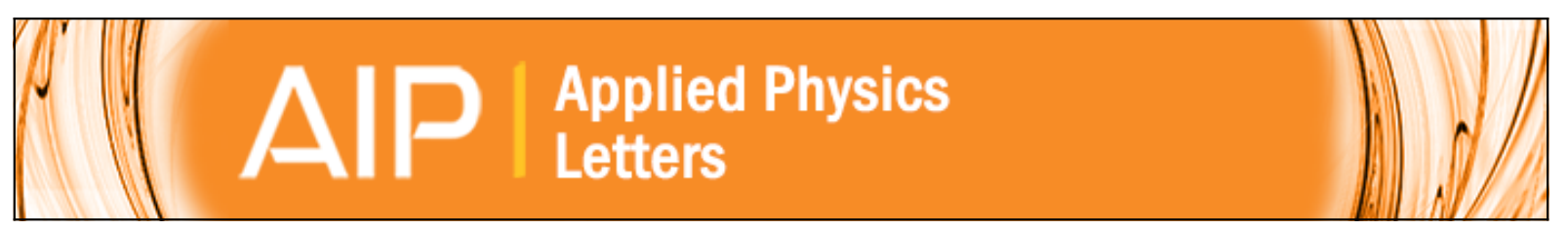

\title{
Thermal stability of magnetoelectric sensors
}

Ying Shen, Junqi Gao, Yaojin Wang, Jiefang Li, and Dwight Viehland

Citation: Applied Physics Letters 100, 173505 (2012); doi: 10.1063/1.4705298

View online: http://dx.doi.org/10.1063/1.4705298

View Table of Contents: http://scitation.aip.org/content/aip/journal/apl/100/17?ver=pdfcov

Published by the AIP Publishing

\section{$\underset{\substack{\text { Anubsurus } \\ \text { And }}}{A}$ Re-register for Table of Content Alerts}




\title{
Thermal stability of magnetoelectric sensors
}

\author{
Ying Shen, Junqi Gao, Yaojin Wang, Jiefang Li, and Dwight Viehland \\ Department of Materials Science and Engineering, Virginia Tech, Blacksburg, Virginia 24061, USA
}

(Received 31 March 2012; accepted 4 April 2012; published online 23 April 2012)

\begin{abstract}
The effect of temperature on the equivalent magnetic noise floor of Metglas- $\mathrm{Pb}(\mathrm{Zr}, \mathrm{Ti}) \mathrm{O}_{3}(\mathrm{PZT})$ laminate magnetoelectric $(\mathrm{ME})$ sensors has been investigated in the temperature range of $-50^{\circ} \mathrm{C}$ to $50^{\circ} \mathrm{C}$. In detail, the parameters that control the noise floor of ME sensors, such as capacitance, $\tan \delta$, and ME charge coefficient, were characterized. The results show the noise floor was thermally stable around $30 \mathrm{pT} / \sqrt{ } \mathrm{Hz}(f=1 \mathrm{~Hz})$ over the studied temperature range. To demonstrate the relative invariance of $\mathrm{ME}$ sensor at different temperatures over the range studied, a simulation based on a noise model was conducted, where the predicted and measured equivalent magnetic noise floors were found to well agree. (C) 2012 American Institute of Physics. [http://dx.doi.org/10.1063/1.4705298]
\end{abstract}

Multiferroic materials, where a coupling between spin and polarization enables a dynamic interaction between these two order parameters, are a broader class of multifunctional materials that combine several useful properties in the same composite to produce a phenomena that is more than just the simple sum of the individual properties of the component phases. $^{1,2}$ For example, magnetoelectric (ME) interactions could have significant cross-coupling effects where polarization can be induced in a magnetically ordered state offering promising applications, in magnetic field sensors and datastorage memory devices. ${ }^{3,4}$

To date, ME laminated composites of magnetostrictive Metglas foils and piezoelectric $\mathrm{Pb}(\mathrm{Zr}, \mathrm{Ti}) \mathrm{O}_{3}$ or $\mathrm{PZT}$ have been widely investigated in various operational modes and optimized for use in magnetic field detection applications. ${ }^{5-7}$ Highly sensitive, room temperature, passive magnetic field sensors have been developed based on ME laminates. Such magnetic sensors have been shown to have noise floors on the order of $\mathrm{pT} / \sqrt{ } \mathrm{Hz}$ for Metglas/PZT foil laminates. The limiting equivalent magnetic noise sources of $\mathrm{ME}$ sensors has been shown to be thermal polarization and DC resistance, both of which are temperature dependent. However, the stability of ME sensors to temperature changes has not yet been determined, in spite of the fact that the sensors need to operate over a broad range of ambient temperatures around $25^{\circ} \mathrm{C}$, when they are used in open environments. It is unknown if the ME voltage coefficient $\left(\alpha_{\mathrm{ME}}\right)$ and equivalent magnetic noise floor are decreased or increased with temperature. Such changes in environment could potentially degrade sensor performance.

Here, we report an investigation of the temperature dependent properties of Metglas/PZT ME sensors in the temperature range of $-50{ }^{\circ} \mathrm{C} \leq T \leq 50{ }^{\circ} \mathrm{C}$. The results show that the equivalent magnetic noise floor is quite resistant to temperature changes, displaying a value of about $30 \mathrm{pT} / \sqrt{\mathrm{Hz}}$ at $f=1 \mathrm{~Hz}$. The sensitivity was found to slightly increase with increasing temperature over the range investigated.

To fabricate ME sensors in a multi push-pull mode configuration, we obtained PZT fibers (CTS, Albuquerque, NM) and Metglas foils (Vitrovac 7600 F, Vitrovac Inc., Hanau, German). The dimensions of the piezoelectric core were $4 \mathrm{~cm} \times 1 \mathrm{~cm} \times 180 \mu \mathrm{m}$, which consisted of a PZT fiber bundle affixed to thin interdigitated (ID) electrodes deposited on
Kapton $^{\circledR}$ sheets by a two-part epoxy. The electrode pattern allowed for a symmetrical polling of the piezoelectric fibers in a back-to-back manner along their length axes. ${ }^{5}$ Three layers of Metglas foils cut into dimensions of $8 \mathrm{~cm} \times 1 \mathrm{~cm} \times 22 \mu \mathrm{m}$ were then attached to the upper and lower surfaces of the Kapton $^{\circledR}$ electrodes using the same epoxy.

Figure 1 shows the ME voltage $\left(\alpha_{\mathrm{ME}}\right)$ and charge $\left(\alpha_{\mathrm{me}}\right)$ coefficients of the Metglas/PZT-fiber sensor as a function of DC magnetic field $H_{\mathrm{dc}}$ at room temperature. Along the longitudinal axis of the laminates, a constant AC magnetic excitation of $H_{\mathrm{ac}}=0.1 \mathrm{Oe}$ was driven at $f=1 \mathrm{kHz}$ by a pair of Helmholtz coils. An electromagnet was used to generate a $H_{\mathrm{dc}}$ along the same axis, and the induced ME voltage signal was directly measured by a lock-in amplifier (Standford Research, SR-850), and the ME charge signal was collected via a charge meter (Kistler type 5015) and measured by the same lock-in amplifier. From this figure, it can be seen that both $\alpha_{\mathrm{ME}}$ and $\alpha_{\mathrm{me}}$ are nearly zero at $H_{\mathrm{dc}}=0$; exhibited a near linear dependence on increasing DC biases for $H_{\mathrm{dc}}<5 \mathrm{Oe}$; reached a maximum value of $\alpha_{\mathrm{me}}=1350 \mathrm{pC} / \mathrm{Oe}$ at an optimal bias of $H_{\mathrm{dc}} \approx 8 \mathrm{Oe}$; and then decreased as $H_{\mathrm{dc}}$ was further increased.

Since the sensitivity of ME composites is mainly limited by the charge noise density $\left(N_{c}\right)$ and the ME charge coefficient, the sensitivity can be estimated as ${ }^{7,8}$

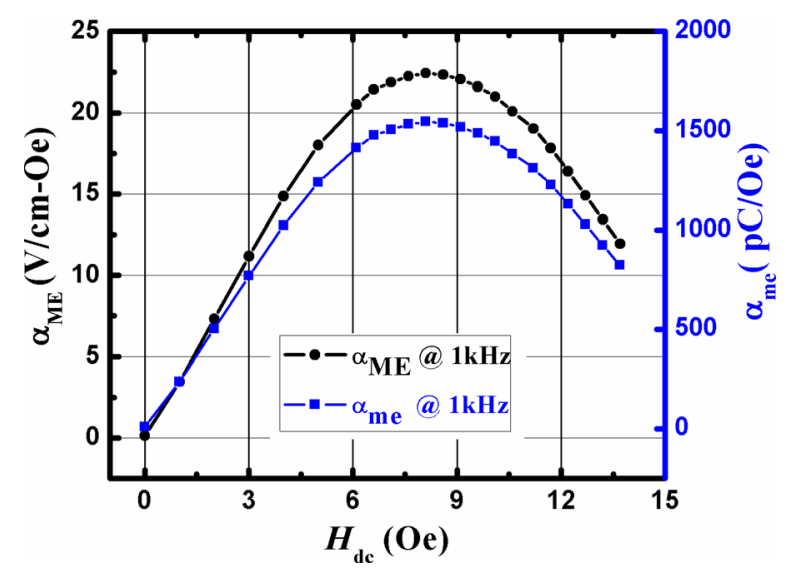

FIG. 1. ME voltage coefficient $\alpha_{\mathrm{ME}}$ (black curve) and ME charge coefficient $\alpha_{\mathrm{me}}$ (blue curve) as a function of dc magnetic field $\mathrm{H}_{\mathrm{dc}}$ for a Metglas/PZT laminate composites at room temperature. 


$$
\text { Sensitivity }\left(\frac{T}{\sqrt{H z}}\right)=\frac{N_{c}\left(\frac{C}{\sqrt{H z}}\right)}{\alpha_{m e} \times 10^{8}\left(\frac{C}{T}\right)} ;
$$

where

$$
N_{c}=\sqrt{\frac{4 k T C \tan \delta}{2 \pi f}+\frac{4 k T}{(2 \pi f)^{2} R}} ;
$$

and where $C$ is the capacitance of the PZT fibers, $\tan \delta$ is the dielectric loss factor of the piezoelectric core ID electrode layer, $R$ is the DC resistance of the PZT core composite, $k$ is the Boltzmann constant $\left(1.38 \times 10^{-23} \mathrm{~J} / \mathrm{K}\right)$, and $T$ is the temperature in Kelvin. This equation can be used to predict the equivalent magnetic noise at different temperatures by putting the temperature-dependent values of $C, \tan \delta$ and $\alpha_{\mathrm{me}}$ into Eq. (1). It is then straightforward to devise an experimental method by which to measure the sensitivity and equivalent magnetic noise of a ME sensor at different temperatures. Unfortunately, previous reports have focused on room temperature operations. Following the approached outline above, we can experimentally and theoretically study the temperature dependent performance.

We characterized temperature dependence of the capacitance, dielectric loss factor, and ME charge coefficient. The ME laminate sensor was placed into a small cooler with dry ice to reduce the inner temperature to $-50{ }^{\circ} \mathrm{C}$. The cooler was mounted on a zero-Gauss, vibration isolation chamber. The temperature was slowly increased from $-50^{\circ} \mathrm{C}$ to $50^{\circ} \mathrm{C}$, while the dielectric constant and loss factor were measured. Figure 2(a) shows $C$ and $\tan \delta(f=1 \mathrm{kHz})$ as a function of temperature, which was measured by an impedance analyzer (Agilent 4294 A). The capacitance increased modestly with increasing temperature from $589 \mathrm{pF}$ $\left(T=-50^{\circ} \mathrm{C}\right)$ to $=659.8 \mathrm{pF}\left(T=50^{\circ} \mathrm{C}\right)$. However, the value of $\tan \delta$ was found to be relatively constant at about 0.01 over the temperature range studied, although at $50{ }^{\circ} \mathrm{C}$ the tan $\delta$ was found to be slightly higher. These $C$ and $\tan \delta$ data are in agreement with previously reported results for the temperature-dependent properties of PZT-based piezoelectric ceramics. For example, PZT-5 A ceramics posses a steady increase in $C$ with temperature and $\tan \delta$ is nearly independent of temperature between $-50{ }^{\circ} \mathrm{C} \leq T \leq 50{ }^{\circ} \mathrm{C}$. ${ }^{9}$

The ME laminates were then integrated into an EMI shielded box with permanent magnets attached at either end of the laminate to provide a DC magnetic biases. Low noise operational amplifier detection circuits were fabricated according to Ref. 10 and assembled into a unit housing with battery support. The sensor detection unit was designed to operate over the frequency bandwidth of $0.6-10 \mathrm{~Hz}$, with a gain factor of $1 \mathrm{~V} / \mathrm{pC}$. Figure 2(b) shows the ME charge coefficient of the ME sensor unit as a function of temperature. The value of $\alpha_{\mathrm{me}}$ at various temperatures was obtained previously by measuring the output signal of the ME sensor at different temperatures and then by computing the amplitude ratio between room temperature and the temperature dependent signals. By employing the ratios with respect to room temperature $(1350 \mathrm{pC} / \mathrm{Oe})$, the $\mathrm{ME}$ charge coefficients at other temperatures were then calculated. During measurement of the output signal, the coil (i.e., controlled source)
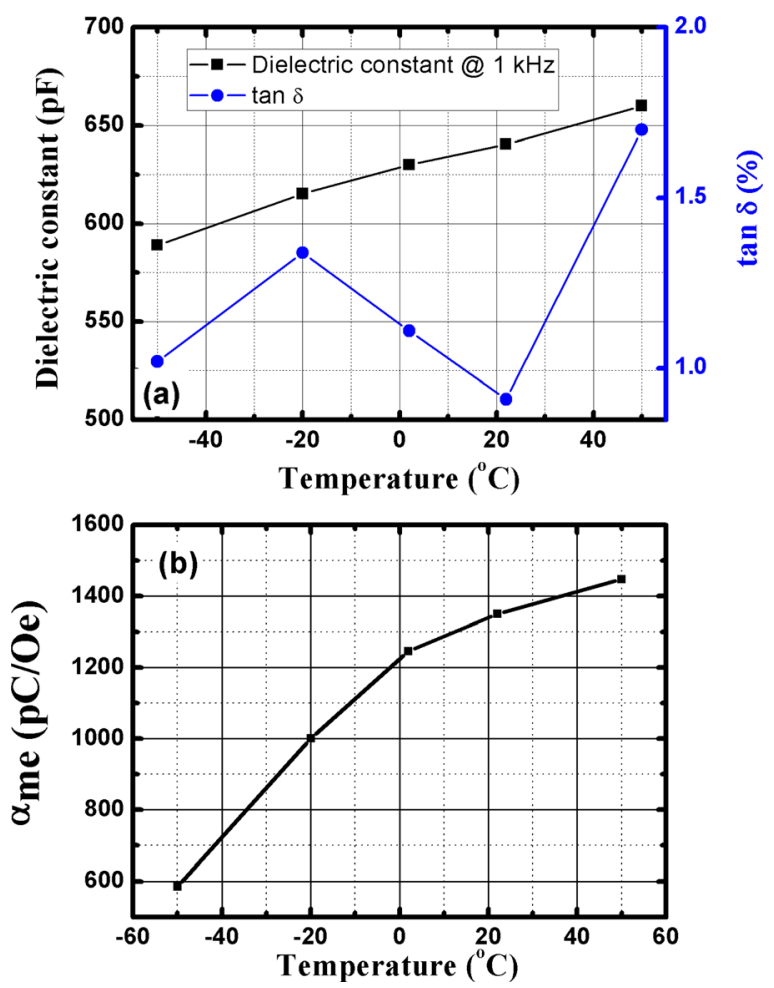

FIG. 2. (a) Capacitance (black curve) and dielectric loss factor (blue curve) as a function of temperature. Measurements were made at $1 \mathrm{kHz}$. (b) ME charge coefficient as a function of temperature.

was located above the cooler shield, centered on the sensor along the longitudinal direction of the laminate. The output signal in response to a constant $\mathrm{AC}$ signal was measured using a dynamic signal analyzer while driving the coil at a frequency of $f=1 \mathrm{~Hz}$ via a lock-in amplifier. Details of the measurement can be found in Ref. 6. In Figure 2(b), one can clearly see with increasing temperature between $-50 \leq T \leq 50^{\circ} \mathrm{C}$ that the ME sensor unit gain factor $\alpha_{\mathrm{me}}$ increased slightly, which can be attributed to changes in the piezoelectric charge coefficient $\left(\mathrm{d}_{33}\right){ }^{9}$

Next, we predicted the ME sensor units equivalent magnetic noise floor as a function of temperature using Eq. (1). Figure 3 shows the simulated equivalent magnetic noise at different temperatures in the frequency range of $1<f<10 \mathrm{~Hz}$. The values of $C, \tan \delta, R_{\mathrm{dc}}$, and $\alpha_{\mathrm{me}}$ at different temperatures

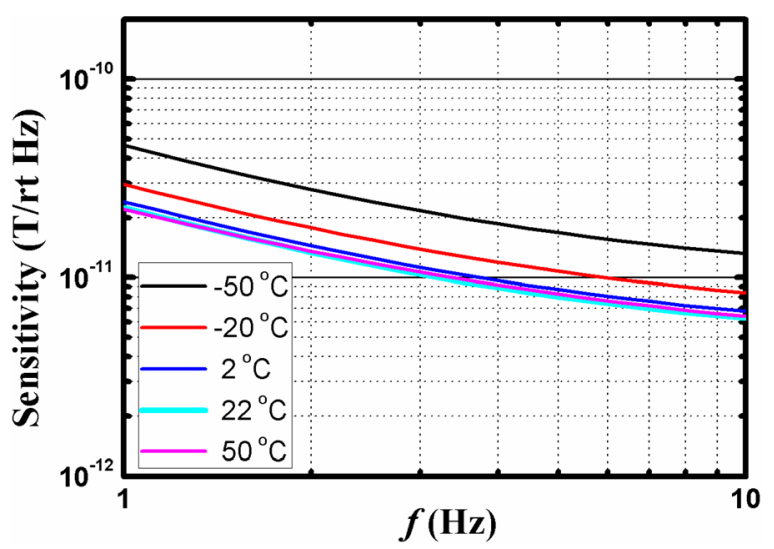

FIG. 3. Predicted equivalent magnetic noise of the ME sensor unit as a function of temperature over the bandwidth of $1<f<10 \mathrm{~Hz}$. The simulations were derived from Eq. (1). 


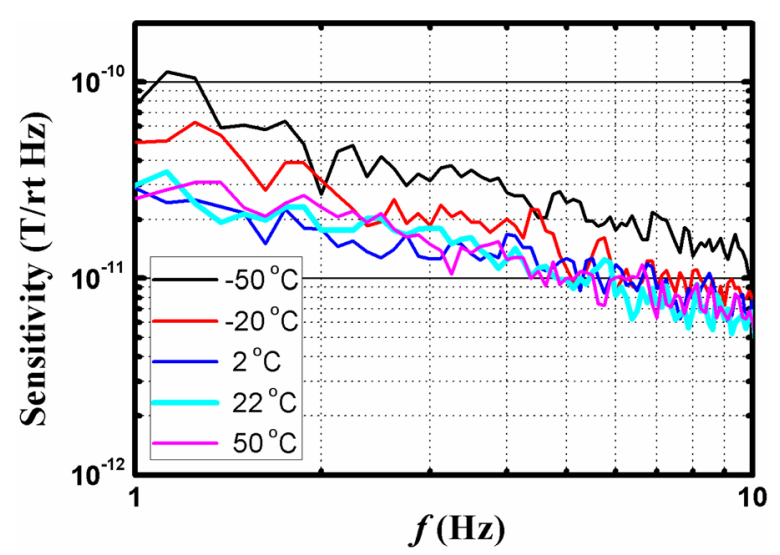

FIG. 4. Measured equivalent magnetic noise spectra for a ME sensor unit at various temperatures of $-50^{\circ} \mathrm{C}$ (black), $-20^{\circ} \mathrm{C}$ (red), $2{ }^{\circ} \mathrm{C}$ (blue), $22^{\circ} \mathrm{C}$ (cyan), and $50^{\circ} \mathrm{C}$ (pink). Please note that these measurements were done in a zero-Gauss vibration isolated chamber, in the absence of any intentional excitation.

were pre-measured (see Figures 2(a) and 2(b)) and put into the noise model. In the noise model, the dominate noise source was assumed to be thermal polarization and DC resistance.

Finally, Figure 4 shows the measured equivalent magnetic noise floor of a $\mathrm{ME}$ sensor unit over the frequency range of $1<f<10 \mathrm{~Hz}$ at the different temperatures evaluated. These measurements were made by directly connecting the sensor unit to a dynamic signal analyzer (Stanford Research, SR-785). The data reveal that the equivalent magnetic noise floor was relatively invariant to temperature, having a value of $30 \mathrm{pT} / \sqrt{\mathrm{Hz}}$ at $f=1 \mathrm{~Hz}$ between $2{ }^{\circ} \mathrm{C}$ and $50^{\circ} \mathrm{C}$. At lower temperatures, the equivalent magnetic noise floor increased to $50 \mathrm{pT} / \sqrt{\mathrm{Hz}}\left(-20^{\circ} \mathrm{C}\right)$ and $75 \mathrm{pT} / \sqrt{\mathrm{Hz}}$ $\left(-50^{\circ} \mathrm{C}\right)$. Comparisons of Figures 3 and 4 will demonstrate good agreement between predicted and measured noise floor values. These studies reveal that ME laminate sensor units offer a viable magnetometer technology for applications in a real-world environment when temperature changes occur. We find that the low noise floors of ME sensors persist over the range of ambient temperatures that might be expected during an annual thermal cycle.

We have investigated the temperature dependence of the performance of ME laminate sensors consisting of Metglas foils bonded to a PZT core. The sensitivity of the sensors to small changes in AC magnetic fields and the equivalent magnetic noise floor were studied over the temperature range of $-50{ }^{\circ} \mathrm{C} \leq T \leq 50{ }^{\circ} \mathrm{C}$. Our results demonstrate that the performance of the sensor was quite stable to temperature changes. The equivalent magnetic noise floor was found to be nearly constant at $30 \mathrm{pT} / \sqrt{ } \mathrm{Hz}(f=1 \mathrm{~Hz})$ over the temperature range investigated. Furthermore, we have applied a noise model to predict the temperature dependent behavior based on input parameters of the $C, \tan \delta$, and $R_{\mathrm{dc}}$. We note that good agreement was found between predicted and measured noise floors.

This work was sponsored by the Office of Naval Research.

${ }^{1}$ W. Eerenstein, N. D. Mathur, and J. F. Scott, Nature 442 (7104), 759-765 (2006).

${ }^{2}$ S. W. Cheong and M. Mostovoy, Nature Mater. 6, 13-20 (2007).

${ }^{3}$ Y. H. Chu, L. W. Martin, M. B. Holcomb, M. Gajek, S. J. Han, Q. He, N. Balke, C. H. Yang, D. Lee, W. Hu, Q. Zhan, P. L. Yang, A. FraileRodriguez, A. Scholl, S. X. Wang, and R. Ramesh, Nature Mater. 7 (6), 478-482 (2008).

${ }^{4}$ C. Ederer and N. A. Spaldin, Nature Mater. 3, 849-851 (2004).

${ }^{5}$ J. Das, J. Gao, Z. Xing, J. F. Li, and D. Viehland, Appl. Phys. Lett. 95 (9), 092501 (2009).

${ }^{6}$ J. Gao, J. Das, Z. Xing, J. Li, and D. Viehland, J. Appl. Phys. 108(8), 084509 (2010).

${ }^{7}$ Y. Wang, D. Gray, D. Berry, J. Gao, M. Li, J. Li, and D. Viehland, Adv. Mater. 23(35), 4111-4114 (2011).

${ }^{8}$ Z. Xing, J. Li, and D. Viehland, Appl. Phys. Lett. 91(18), 182902 (2007).

${ }^{9}$ M. W. Hooker, NASA/CR 208708 (1998).

${ }^{10}$ Z. P. Xing, J. Y. Zhai, S. X. Dong, J. F. Li, D. Viehland, and W. G. Odendaal, Meas. Sci. Technol. 19(1), 015206 (2008). 